\title{
CD4+T-lymphocyte subsets in nonobese children with obstructive sleep apnea syndrome
}

\author{
Jin Ye', Hui Liu², Peng Li' ${ }^{1}$ Zhuang-gui Chen³ ${ }^{3}$ Ge-hua Zhang' ${ }^{1}$ Qin-tai Yang ${ }^{1}$ and Yuan Li ${ }^{1}$
}

\begin{abstract}
BACKGROUND: To characterize the distribution of both tonsillar and circulating CD4 ${ }^{+}$-lymphocyte subsets, and to explore their clinical relevance in nonobese children with obstructive sleep apnea syndrome (OSAS).
\end{abstract}

METHODS: A total of 53 children who underwent tonsillectomy for either OSAS ( $n=25)$ or primary snoring (PS, $n=28$ ) were prospectively enrolled. Nineteen healthy children without any symptoms were recruited as controls. We quantified the frequencies of $\mathrm{CD}^{+}{ }^{+}$-lymphocyte subpopulations using flow cytometry, serum-related cytokines using enzyme-linked immunosorbent assay, and key transcription factors using quantitative polymerase chain reaction (qPCR).

RESULTS: Tonsillar distributions of CD4 ${ }^{+}$T-lymphocyte subsets were comparable in the OSAS and PS subjects. The peripheral Th17/Treg ratio was positively correlated to severity as measured by apnea/hypopnea index (AHI), serum C-reactive protein and hypoxia-inducible factor- $1 \alpha$ mRNA in the OSAS children $(P<0.05)$. And AHI was independently associated with the peripheral Th17/Treg ratio $(P<0.05)$. Furthermore, the response to surgery was associated with a significant reversal of the Th17/Treg imbalance and a concomitant relief of the proinflammatory profile in the OSAS subjects.

CONCLUSION: Pediatric OSAS was associated with an altered Th17:Treg balance toward Th17 predominance. The changes in lymphocytic phenotypes that correlated with recurrent intermittent hypoxia in sleep apnea may contribute to the variance in systemic inflammation and downstream morbidities of pediatric OSAS.

$S^{t}$ eep-disordered breathing (SDB) represents a spectrum of breathing disorders that range from primary snoring (PS) to obstructive sleep apnea syndrome (OSAS). A dysfunction in neuromotor control is thought to be the underlying cause of airway collapse during sleep in children, but adenotonsillar hypertrophy further narrows the airway and exacerbates OSAS. To date, the diagnosis of pediatric OSAS totally relies on overnight polysomnography (PSG). There are inherent difficulties in clinical discriminating OSAS from PS, especially in children possessing analogous symptoms and physical signs (1).
A central mechanism that mediates local and systemic inflammation in OSAS involves the intermittent nocturnal hypoxemia that may produce oxygen-free radicals through the phenomenon of the intermittent hypoxia and reoxygenation (IHR) (2). As the characteristic feature of OSAS, recurrent cycles of IHR can promote an extensive activation of various inflammatory cells, as well as specifically induce the hypoxia-sensitive transcription factors hypoxia-inducible factor-1(HIF-1) and the subsequent activation of inflammatory pathways. It is proved that HIF-1-dependent inflammation has a principal role in the promoting varied cardiovascular consequences in OSAS patients (3).

Disturbances in the differentiation and function of $\mathrm{CD} 4^{+} \mathrm{T}$ lymphocyte subsets (Th1, Th2, Th17, Th22, and Treg) represent an indicator of the host immune response in human cancer, inflammatory, and autoimmune diseases $(4,5)$. We have demonstrated a positive correlation of peripheral Th17/Treg ratio with the incidence of apnea/hypopnea index (AHI) in OSAS adults (6). HIF-1 is proved as a check point of Th17/Treg polarization (7). We conjecture that the distinctive repetitions of IHR cycle in OSAS children directly bring about the disproportion of $\mathrm{CD} 4^{+} \mathrm{T}$ lymphocyte subsets through activating HIF-1. Therefore, such an immune imbalance may serve as a reliable screening index for the clinical diagnosis of pediatric OSAS.

To confirm our hypothesis, we started with characterizing the constitution of $\mathrm{CD} 4^{+} \mathrm{T}-$ lymphocyte lineages in both hypertrophied tonsils and circulation for OSAS children as compared with PS subjects. Next, the potential relevance of lymphocyte patterns with OSAS severity, HIF-1, and inflammatory biomarkers were evaluated. Furthermore, we performed the follow-up visits to assess the effect of surgery on the distribution of circulating $\mathrm{CD} 4^{+} \mathrm{T}-$-lymphocyte subsets.

\section{RESULTS}

\section{Basic Characteristics}

A total of 53 nonobese children underwent tonsillectomy (with or without adenoidectomy) for obstructive adenotonsillar hypertrophy was included in the final analyses. Twenty-five children were confirmed to be OSAS (OSAS group), and 28 cases were PS group according to an overnight PSG. Nineteen

\footnotetext{
'Sleep Disorders Centre and Department of Otolaryngology-Head and Neck Surgery, The Third Affiliated Hospital of Sun Yat-sen University, Guangzhou, China; ${ }^{2}$ Department of Internal Medicine, Division of Pulmonary and Critical Care, The Third Affiliated Hospital of Sun Yat-sen University, Guangzhou, China; ${ }^{3}$ Department of Pediatrics, The Third Affiliated Hospital of Sun Yat-sen University, Guangzhou, China. Correspondence: Jin Ye (yejin_sums@aliyun.com) 
matched children were enrolled as healthy controls (HC group). The baseline parameters of the three groups are summarized in Table 1. All participants were Chinese. There was no obvious difference in tonsillar size, EES, or OSA-18 score between the PS group and the OSAS group before AT. The mean of obstructive AHI was significantly higher in the OSAS group $(34.76 \pm 15.28)$ than the PS group $(0.76 \pm 0.21)$. Three subjects were classified with mild OSAS, 5 subjects were moderate, and 17 subjects were severe according to preoperative PSG.

\section{Lymphocytic Distribution in Tonsils and Circulation}

No difference between girls and boys was emerged $(P>0.05)$. As shown in Table 2, the distribution of tonsillar $\mathrm{CD}^{+} \mathrm{T}$ lymphocyte subsets was comparable between PS and OSAS groups $(P>0.05$ for all). In contrast, the percentages of peripheral Th1, Th17, and Th22 lymphocytes were increased and Tregs was significantly decreased in the OSAS group compared to the PS and HC groups (Table 3; Figures 1 and 2). As a result, the ratios of Th17/Treg, Th1/Treg, and Th22/Treg were obviously higher in OSAS subjects in contrast to other two groups. In addition, there were no differences between the counts of lymphocyte subsets in the tonsils and the same subsets in the peripheral blood (Tables 2 and 3 ).

The quantification of representative cytokines by enzymelinked immunosorbent assay (ELISA) and transcription factors by qPCR verified the results of flow cytometry. For instance, the peripheral Th17 frequency in all participants was positively correlated with plasma concentrations of IL-17 ( $r=0.538, P=0.006)$ and the expression of ROR $\gamma \mathrm{t}$ mRNA in peripheral blood mononuclear cells (PBMCs) $(r=0.436$, $P=0.011)$. Similar linear correlations were confirmed with other lymphocyte subsets. In brief, it turned out to be good consistency between three methods.

\section{Circulating Th17/Treg Imbalance Characterized OSAS}

In the OSAS children, the proportion of various lymphocyte subtypes expressed, either alone or as ratios, were not

Table 1. Demographic and clinical characteristics of studied subjects at baseline

\begin{tabular}{|c|c|c|c|}
\hline Baseline parameters & $\mathrm{HC}$ & PS & OSAS \\
\hline Subjects, $n$ & 19 & 28 & 25 \\
\hline Age, mean (range) & $6.63 \pm 2.71$ & $6.81 \pm 2.33$ & $6.45 \pm 2.84$ \\
\hline Male, $n(\%)$ & $12(63.16)$ & $17(60.71)$ & $16(64.00)$ \\
\hline BMI z score, mean (range) & $1.25 \pm 0.47$ & $1.19 \pm 0.52$ & $1.28 \pm 0.64$ \\
\hline Tonsillar size & $0.53 \pm 0.20$ & $3.13 \pm 1.04$ & $3.05 \pm 0.95$ \\
\hline AHI, episodes/h & $0.38 \pm 0.20$ & $0.76 \pm 0.31$ & $34.76 \pm 15.28^{*}$ \\
\hline Nadir $\mathrm{SaO}_{2}(\%)$ & $95.78 \pm 4.88$ & $94.34 \pm 3.92$ & $72.47 \pm 16.83^{*}$ \\
\hline $\mathrm{TSpO}_{2}<90 \%(\%)$ & $1.69 \pm 0.36$ & $2.28 \pm 0.86$ & $9.83 \pm 5.36^{*}$ \\
\hline OSA-18 scores & - & $78.61 \pm 23.83$ & $80.7 \pm 21.52^{*}$ \\
\hline ESS & - & $18.02 \pm 11.54$ & $16.41 \pm 3.67^{*}$ \\
\hline
\end{tabular}

Values are expressed as mean \pm SD.

$\mathrm{HC}$, healthy controls; OSAS, obstructive sleep apnea syndrome; PS, primary snoring $* P<0.05$, HC vs. OSAS. associated with tonsillar size, OSA-18 or ESS score (Table 4). Instead, the circulating Th17/Treg ratio was correlated with AHI $(r=0.716, P<0.001)$ and proinflammatory markers including hypersensitive C-reactive protein (hsCRP) $(r=0$. 473, $P=0.017)$ and HIF- $1 \alpha(r=0.761, P<0.001$; Figure 3$)$. Moreover, HIF-1 $\alpha$ mRNA expression in PBMCs was positively correlated with AHI $(r=0.584, P<0.001)$.

As for PS group, the circulating Th17/Treg ratios were not significantly correlated with AHI $(r=0.104, P=0.061)$ or proinflammatory markers, including hsCRP $(r=0.137, P=0.143)$ and HIF- $1 \alpha(r=0.110, P=0.174)$. No correlation mentioned above was identified (data not shown).

Next, we performed stepwise regression analyses to further examine independent predictors of circulating Th17/Treg ratio in the OSAS children (Table 5). The Th17/Treg ratio in the initial univariate analysis exhibited a significant correlation with AHI $(P=0.017)$ and HIF-1 $\alpha(P=0.035)$ but not tonsillar grade $(P=0.058)$.The circulating Th17/Treg ratio in the multiple regression analysis was only positively associated with AHI $(P=0.006)$ after controlling for potential confounding factors.

\section{Changes in Lymphocytic Proportions and Serum Inflammatory Cytokines After Adenotonsillectomy in OSAS Children}

A total of 43 children (20 children with PS and 23 cases with OSAS) completed the follow-up visit 6 mo after surgery, while the remaining 10 children completed clinical evaluations over the telephone. For the 23 OSAS children, an apparent decrease in OSA-18 $(80.7 \pm 21.52$ vs. $30.6 \pm 14.9, P<0.001)$ and ESS scores $(16.41 \pm 3.67$ vs. $4.25 \pm 1.63, P<0.001)$ were identified. All patients showed significant reductions in OSAS severity as illustrated by AHI (34.76 \pm 15.28 vs. $3.04 \pm 1.82, P<0.05)$.

The changes in the distribution of $\mathrm{CD} 4^{+} \mathrm{T}$ subsets and corresponding cytokines and transcription factors are presented in Table 3 and Figures 4 and 5. The peripheral frequencies

Table 2. Descriptive statistics of tonsillar lymphocyte distribution in sleep-disordered breathing children underwent adenotonsillectomy

\begin{tabular}{lcc}
\hline Tonsillar distribution & PS & OSAS \\
\hline Subjects, $n$ & 28 & 25 \\
Flow cytometry & & \\
Th1 (\% CD4) & $21.73 \pm 7.56$ & $23.48 \pm 8.32$ \\
Th2 (\% CD4) & $12.52 \pm 3.49$ & $13.72 \pm 6.43$ \\
Th17 (\% CD4) & $6.38 \pm 0.79$ & $5.84 \pm 1.02$ \\
Th22 (\% CD4) & $5.23 \pm 0.83$ & $4.94 \pm 1.13$ \\
Treg (\% CD4) & $2.72 \pm 1.18$ & $2.05 \pm 1.26$ \\
RT-qPCR & & \\
T-bet/GADPH & $3.76 \pm 1.74$ & $4.68 \pm 1.53$ \\
GATA-3/GADPH & $4.46 \pm 1.25$ & $5.09 \pm 1.27$ \\
RORyt/GADPH & $2.58 \pm 1.26$ & $2.12 \pm 1.36$ \\
AhR/GADPH & $2.21 \pm 1.30$ & $2.53 \pm 1.61$ \\
FOXP3/GADPH & $1.04 \pm 0.57$ & $1.44 \pm 0.45$ \\
\hline P>0.05 for all comparison. & & \\
OSAS, obstructive sleep apnea syndrome; PS, primary snoring.
\end{tabular}


of Th1, Th22, and Th17 cells were apparently decreased and the peripheral Treg percentage was obviously increased after surgery in the OSAS subjects $(P<0.05$, Table 3$)$. Coincident results were confirmed with corresponding cytokines and transcription factors. Consequently, the Th17/Treg ratio was significantly decreased after surgery ( $1.14 \pm 0.56$ vs. $0.22 \pm 0.11$, $P<0.05$, Figure 5).

Before surgery, HIF- $1 \alpha$ and hsCRP expression were both markedly increased in OSAS group comparing with PS and HC subjects (Table 3). But a positive correlation between hsCRP concentration and HIF- $1 \alpha$ mRNA expression was only demonstrated in the OSAS children $(r=0.463, P=0.023$, Figure 3), but not in the PS group $(r=0.285, P=0.074)$ or the HC group $(r=0.193, P=0.107)$. As shown in Figure 5, AT could normalize the levels of circulating HIF- $1 \alpha$ and hsCRP in OSAS children. In a word, the peripheral Th17/Treg ratio was markedly reduced after surgery and accompanied by a parallel amelioration in OSAS severity and the proinflammatory state.

\section{DISCUSSION}

Here, we showed that the peripheral Th17/Treg ratio was positively correlated with the incidence of IHR, namely AHI in OSAS children. Moreover, the correction of the Th17/Treg imbalance was parallel to the elimination of obstruction and hypoxia after adenotonsillectomy. It further demonstrated that such a disproportion of $\mathrm{CD} 4^{+} \mathrm{T}$ - lymphocyte subsets was directly derived from OSAS. In this sense, it can serve as a clinic indicator of OSAS.

\section{The Imbalance of $\mathrm{CD}^{+} \mathrm{T}$-Lymphocyte Subsets}

It should be noted that only PS children who underwent adenotonsillectomy due to obvious tonsillar hypertrophy were eligible for our analyses. The PS and OSAS children were carefully matched for tonsillar sizes and obstructive symptoms. The similar extent of local inflammation may explain the comparable distribution pattern of tonsilar lymphocytes in two groups. Consistently, Verhulst et al. (8) found that the airway inflammation was not appreciably different in PS and OSAS children. Indeed, the enhanced inflammatory events in local tonsils could be triggered and maintained by snoring and the associated vibration frequencies, with recurring upper airway collapse that promotes soft-tissue damage. In this sense, the extent of lymphocyte activation in hypertrophied tonsils can hardly discriminate pediatric OSAS from other types of SDB.

In contrast, the circulating formation of lymphocyte subsets exhibited a closer relevance with the proinflammatory status

Table 3. Systemic pattern of lymphocyte subsets before and after adenotonsillectomy

\begin{tabular}{|c|c|c|c|c|c|}
\hline \multirow[b]{2}{*}{ Systematic parameters } & \multirow[b]{2}{*}{$\mathrm{HC}$} & \multicolumn{2}{|c|}{ PS } & \multicolumn{2}{|c|}{ OSAS } \\
\hline & & Presurgery & Postsurgery & Presurgery & Postsurgery \\
\hline Subjects, $n$ & 19 & 28 & 20 & 25 & 23 \\
\hline Th1 (\% CD4) & $11.73 \pm 3.59$ & $17.32 \pm 4.20^{\ddagger}$ & $12.63 \pm 4.36^{\S}$ & $25.07 \pm 6.71^{*,+, \neq}$ & $14.63 \pm 3.78^{* *}$ \\
\hline Th2 (\% CD4) & $3.62 \pm 1.20$ & $3.23 \pm 0.96$ & $3.78 \pm 1.48$ & $3.49 \pm 1.21$ & $3.50 \pm 1.15$ \\
\hline Th22 (\% CD4) & $0.92 \pm 0.63$ & $2.00 \pm 0.74$ & $0.95 \pm 0.44$ & $3.29 \pm 1.02^{*, t, \neq}$ & $1.17 \pm 0.38^{* *}$ \\
\hline Treg (\% CD4) & $9.36 \pm 3.80$ & $5.81 \pm 2.83^{\ddagger}$ & $7.54 \pm 2.19^{\S}$ & $3.62 \pm 0.64^{*, t, \neq}$ & $6.35 \pm 1.28^{* *}$ \\
\hline \multicolumn{6}{|l|}{ qPCR } \\
\hline T-bet/GADPH & $3.64 \pm 0.37$ & $4.45 \pm 1.19^{\ddagger}$ & $3.23 \pm 0.43^{\S}$ & $5.67 \pm 1.32^{*}$ & $3.33 \pm 0.39^{* *}$ \\
\hline GATA-3/GADPH & $2.35 \pm 0.46$ & $2.47 \pm 0.63$ & $1.91 \pm 0.33$ & $2.39 \pm 0.51$ & $1.98 \pm 0.42$ \\
\hline HIF-1 $\alpha /$ GADPH & $6.58 \pm 2.84$ & $8.06 \pm 6.28$ & $7.38 \pm 3.46$ & $26.16 \pm 10.41^{*,+}$ & $9.87 \pm 4.76^{* *}$ \\
\hline \multicolumn{6}{|l|}{ ELISA (pg/ml) } \\
\hline hsCRP & $22.75 \pm 9.46$ & $70.36 \pm 20.84^{\ddagger}$ & $36.37 \pm 10.44^{\S}$ & $117.48 \pm 21.12^{*,+}$ & $37.78 \pm 16.19^{* *}$ \\
\hline $\mathrm{IFN} \gamma$ & $26.01 \pm 13.54$ & $38.70 \pm 14.32$ & $22.74 \pm 9.32^{\S}$ & $42.84 \pm 16.61^{*,+}$ & $29.69 \pm 9.99 * *$ \\
\hline IL-4 & $33.24 \pm 15.69$ & $30.74 \pm 13.78$ & $32.91 \pm 14.38$ & $36.72 \pm 16.58$ & $34.02 \pm 11.58$ \\
\hline IL-17 & $38.18 \pm 21.63$ & $66.09 \pm 21.45^{\ddagger}$ & $40.57 \pm 19.30^{\S}$ & $88.36 \pm 14.96^{*,+}$ & $44.95 \pm 14.69^{* *}$ \\
\hline IL-22 & $31.25 \pm 19.14$ & $35.74 \pm 20.62^{\ddagger}$ & $34.56 \pm 20.18^{\S}$ & $70.76 \pm 16.43^{*,+}$ & $40.74 \pm 12.46^{* * *}$ \\
\hline TGF- $\beta_{1}$ & $60.31 \pm 28.45$ & $46.38 \pm 24.12$ & $58.92 \pm 21.53^{5}$ & $30.72 \pm 9.44^{*, t}$ & $53.13 \pm 10.49 * *$ \\
\hline
\end{tabular}

Values are expressed as mean \pm SD.

$\mathrm{HC}$, healthy controls; hsCRP, hypersensitive C-reactive protein. OSAS, obstructive sleep apnea syndrome; PS, primary snoring.

${ }^{*} P<0.05$, Control vs. preoperative OSAS. ${ }^{*} P<0.05$, preoperative OSAS vs. postoperative OSAS. ${ }^{+} P<0.05$, preoperative HS vs. preoperative OSAS. ${ }^{\ddagger} P<0.05$, Control vs. preoperative HS.

${ }^{\circledR} P<0.05$, preoperative HS vs. postoperative HS. 


\section{Articles | Ye etal.}
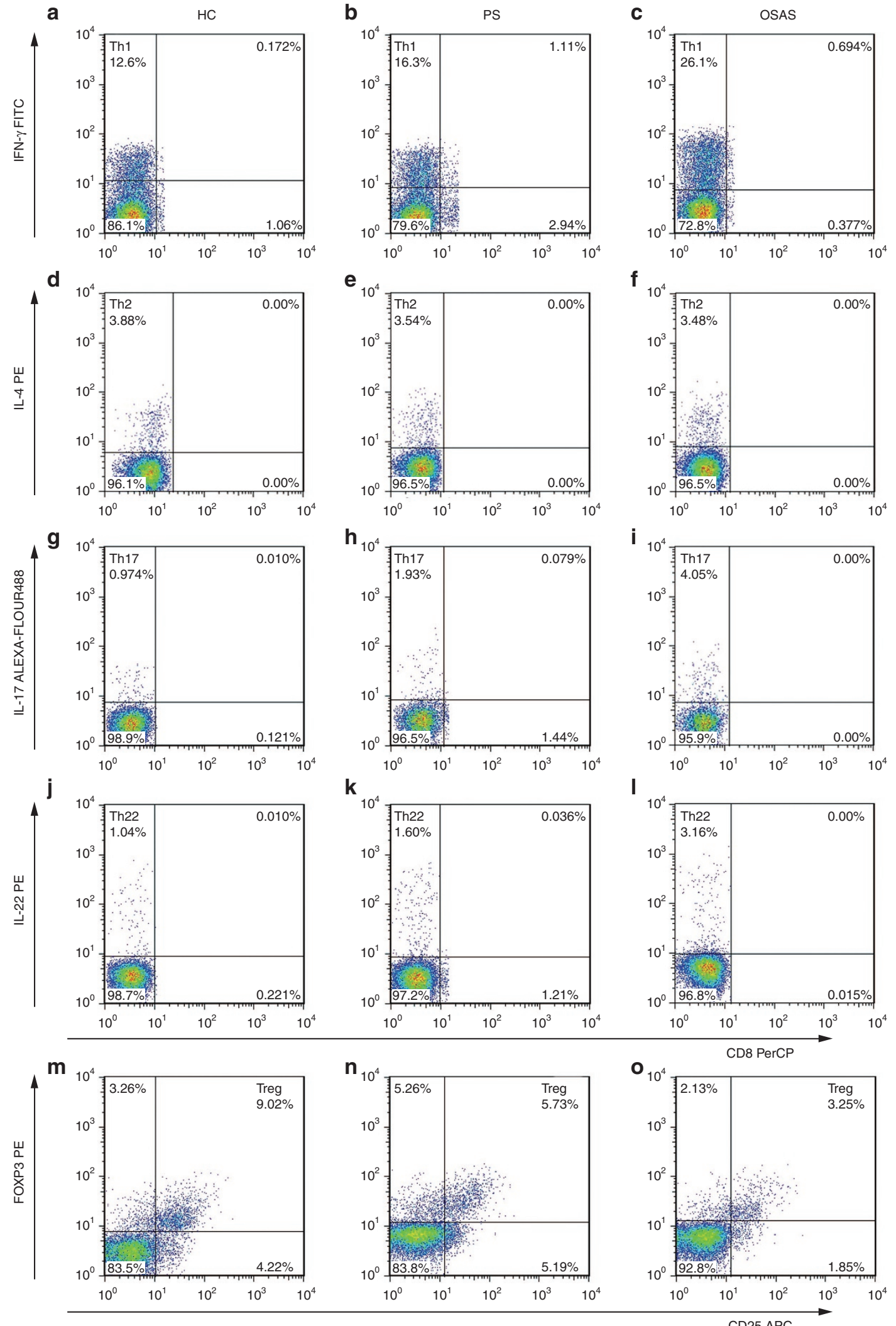

Figure 1. Representative dot plots of circulating CD4+T subpopulations. The distributions of Th1, Th2, Th17, Th22, and Treg cells were measured by FACS in children with OSAS, primary snoring (PS), and healthy controls (HC). Results were expressed as the percentage of single subset in total CD4 ${ }^{+}$cells isolated from PBMCs. CD4 ${ }^{+}$T-lymphocytes were gated as CD3 ${ }^{+} \mathrm{CD} 8^{-}$. Defining the human Th1 (a-c) as CD4 $4^{+} \mathrm{IFN} \gamma^{+}$cells, Th2 (d-f) as CD4 $4^{+} \mathrm{IL}-4^{+}$cells, Th17 (g-i) as CD4+IFN- $\gamma$ IL-22-IL17+ cells, Th22 (j-I) as CD4+IFN- $\gamma$ IL-17-IL-22+ 
a
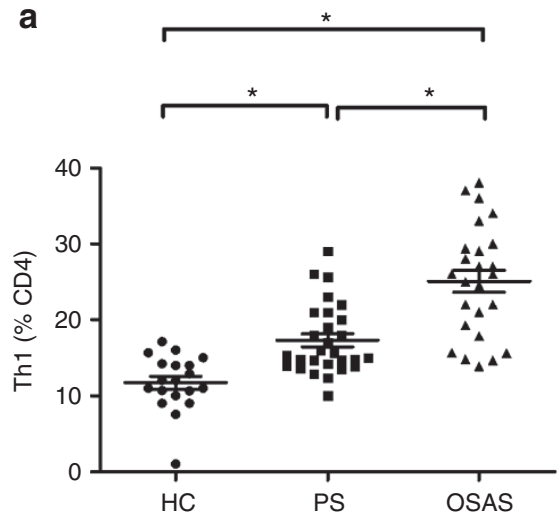

b
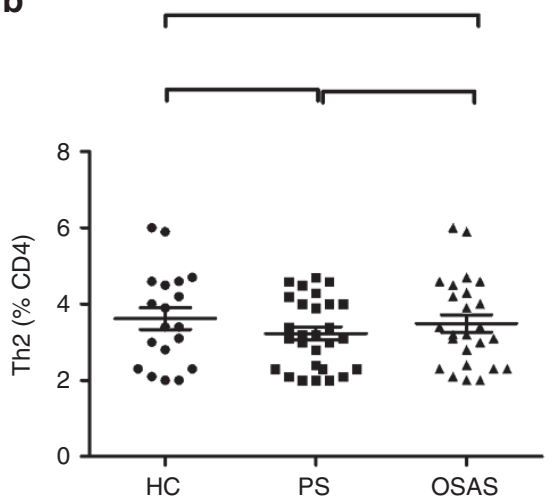

C

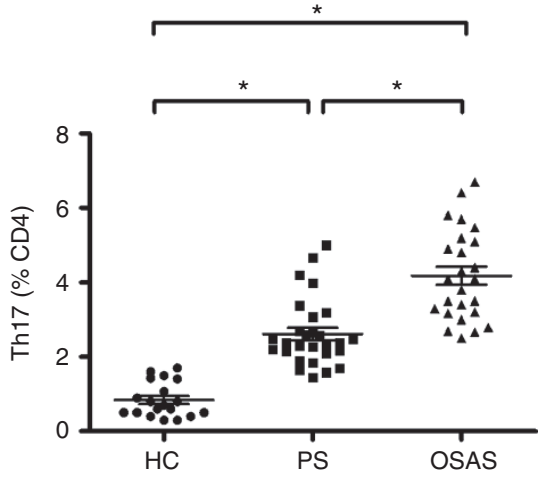

d

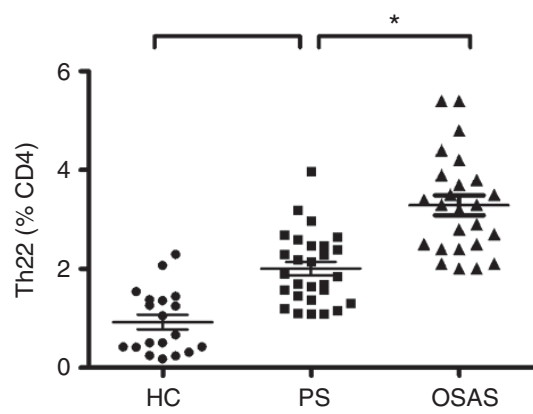

e

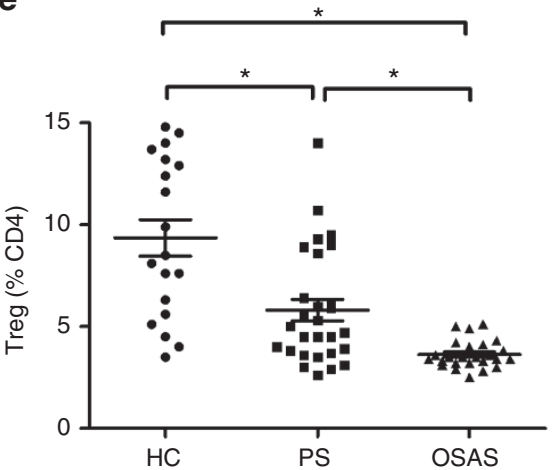

Figure 2. Statistical results for the frequencies of peripheral CD4+T subpopulations. The comparison of peripheral Th1 (a), Th2 (b), Th17 (c), Th22 (d), and Treg (e) cells in OSAS $(n=25)$, PS $(n=28)$, and HC groups $(n=19)$. The horizontal lines represent the median values and error bars represent standard errors of means (SEM). ${ }^{*} P<0.05$.

Table 4. Correlation coefficients of systematic CD4+T subsets distribution with other variables in OSAS children $(n=25)$

\begin{tabular}{|c|c|c|c|c|c|c|c|}
\hline Systematic parameters & $\mathrm{AHI}$ & Nadir $\mathrm{SaO}_{2}(\%)$ & Tonsillar size & OSA-18 & ESS & hsCRP & HIF-1 $\alpha$ \\
\hline Th17 (\% CD4) & 0.273 & 0.189 & 0.235 & 0.039 & 0.114 & 0.305 & 0.237 \\
\hline Th22 (\% CD4) & 0.249 & 0.217 & 0.109 & 0.164 & 0.207 & 0.363 & 0.308 \\
\hline Treg (\% CD4) & -0.112 & 0.106 & 0.278 & 0.205 & 0.246 & -0.234 & -0.319 \\
\hline Th1/Th2 ratio & 0.195 & 0.092 & 0.234 & 0.142 & 0.178 & 0.273 & 0.234 \\
\hline Th17/Treg ratio & $0.687^{*}$ & 0.156 & 0.259 & 0.129 & 0.246 & $0.464^{*}$ & $0.835^{*}$ \\
\hline Th17/Th22 ratio & 0.128 & 0.071 & 0.096 & 0.175 & 0.274 & 0.301 & 0.215 \\
\hline
\end{tabular}

The boldfacing means statistical significance. ${ }^{*} P<0.05$.

AHI, apnea/hypopnea index; ESS, Epworth sleepiness scale; hsCRP, hypersensitive C-reactive protein. OSAS, obstructive sleep apnea syndrome.

a

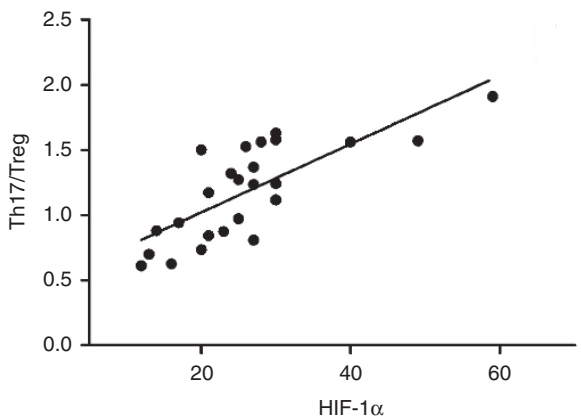

b

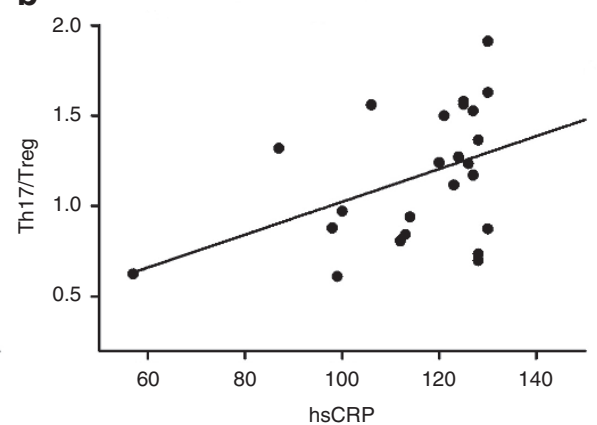

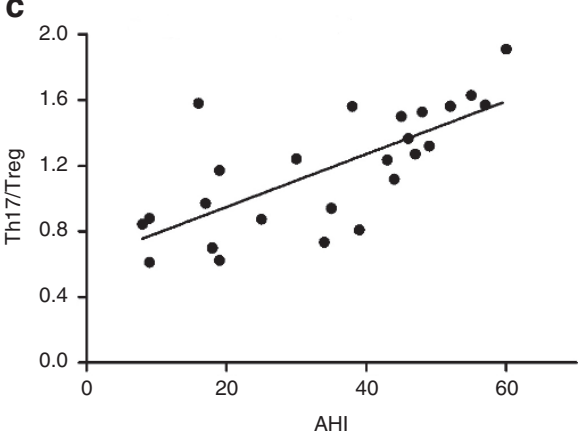

Figure 3. Spearman correlation of circulating Th17/Treg ratio with plasma hsCRP, HIF-1 $\alpha$ mRNA, and the severity as measured by AHI in OSAS children $(n=25)$. All of these data were collected before surgery and each point represents a sample. The circulating Th17/Treg ratio was positively correlated with HIF-1 $\alpha$ (a) mRNA expression in PBMCs $(r=0.761, P<0.001)$, plasma hsCRP (b) concentrations $(r=0.473, P=0.017)$, and presurgical AHI (c) $(r=0.716$, $P<0.001)$, respectively. hsCRP, hypersensitive C-reactive protein. 
in OSAS subjects. The Th17/Treg imbalance is proved to be widely existed in human cancer, inflammatory, and autoimmune diseases (4-6). In consistence with our results in adults with OSAS (6), the imbalanced Th17/Treg ratio was independently correlated with the severity of OSAS. The altered Th17/Treg balance may contribute to the variance in systemic inflammation and downstream morbidities that are associated with OSAS $(3,9)$. The balance or interplay between various immune cells reflects the reciprocity of anti- and proinflammatory driving forces, which serves as a promising clinical assessment predictor.

A positive relationship between circulating Th17 and Th22 frequencies identified in our OSAS children had been demonstrated in various diseases (10-12).This functional synergism of Th2 2 and Th17 cells could lead to a dramatic and persistent inflammation in OSAS. On the other hand, Tan et al. $(13,14)$

Table 5. Univariate and multivariate analyses between Th17/Treg and parameters in OSAS children $(n=25)$

\begin{tabular}{|c|c|c|c|c|c|c|}
\hline \multirow[b]{3}{*}{ Independent variables } & \multicolumn{6}{|c|}{ Systemic Th17/Treg ratio } \\
\hline & \multicolumn{3}{|c|}{ Univariate } & \multicolumn{3}{|c|}{ Stepwise multivariate } \\
\hline & $\beta$ & SE & $P$ value & B & SE & $P$ value \\
\hline Age & 0.031 & 0.024 & 0.140 & - & - & - \\
\hline Sex & 0.165 & 0.042 & 0.121 & - & - & - \\
\hline BMI z score & 0.078 & 0.063 & 0.097 & - & - & - \\
\hline Tonsillar grade & 0.246 & 0.098 & 0.058 & - & - & - \\
\hline $\mathrm{AHI}$ & 0.562 & 0.135 & $0.017^{*}$ & 0.734 & 0.186 & 0.006 \\
\hline HIF-1 $\alpha$ & 0.495 & 0.124 & $0.035^{*}$ & 0.508 & 0.110 & $0.054 *$ \\
\hline
\end{tabular}

The boldfacing means statistical significance. ${ }^{*} P<0.05$.

AHI, apnea/hypopnea index; OSAS, obstructive sleep apnea syndrome. demonstrated that children with OSAS had a lower percentage of Tregs than controls. A declined in frequency and impaired function of Tregs in pediatric OSAS may be associated with a young age (15), sleep fragmentation or sleep deprivation (16), a higher incidence of methylation in iFOXP3 gene (17), and varied phenotypes.

\section{HIF- $1 \alpha$ in OSAS}

Yuan reported an obvious increase in the transcriptional activity of the HIF-1 gene in response to 60 and 120 cycles of IHR but not to fewer cycles (18). Actually, the AHI index represents the mean incidence of IHR cycle per hour throughout the night. Thus, we speculate that HIF-1 expression can only be intensively enhanced when the extent and frequency of IHR increases to a particular point. These may explain the positive correlation between HIF-1 $\alpha$ and AHI identified only in the OSAS subjects but not in the PS group.

Besides an inflammatory cytokine, HIF- $1 \alpha$ is proved to be a critical integrator of metabolic cues that are responsible for the initiation of an adaptive cellular response to hypoxia by orchestrating a Th17/Treg disproportion (19). Here, we detected the potential relationship between HIF- $1 \alpha$ and Th17/Treg disproportion in order to clarify the underlying mechanism of immune imbalance in OSAS. As a result, a positive correlation between them further corroborates our speculation that the repetitive IHR cycles in OSAS lead to HIF- $1 \alpha$ gene activation and subsequently immune dysregulation.

\section{Surgery on the Profile of Lymphocyte Subsets}

Improvements in SDB-related symptoms were accompanied by an apparent abatement of circulating Th17 and Th22 activation and a significant upregulation of Treg percentage in
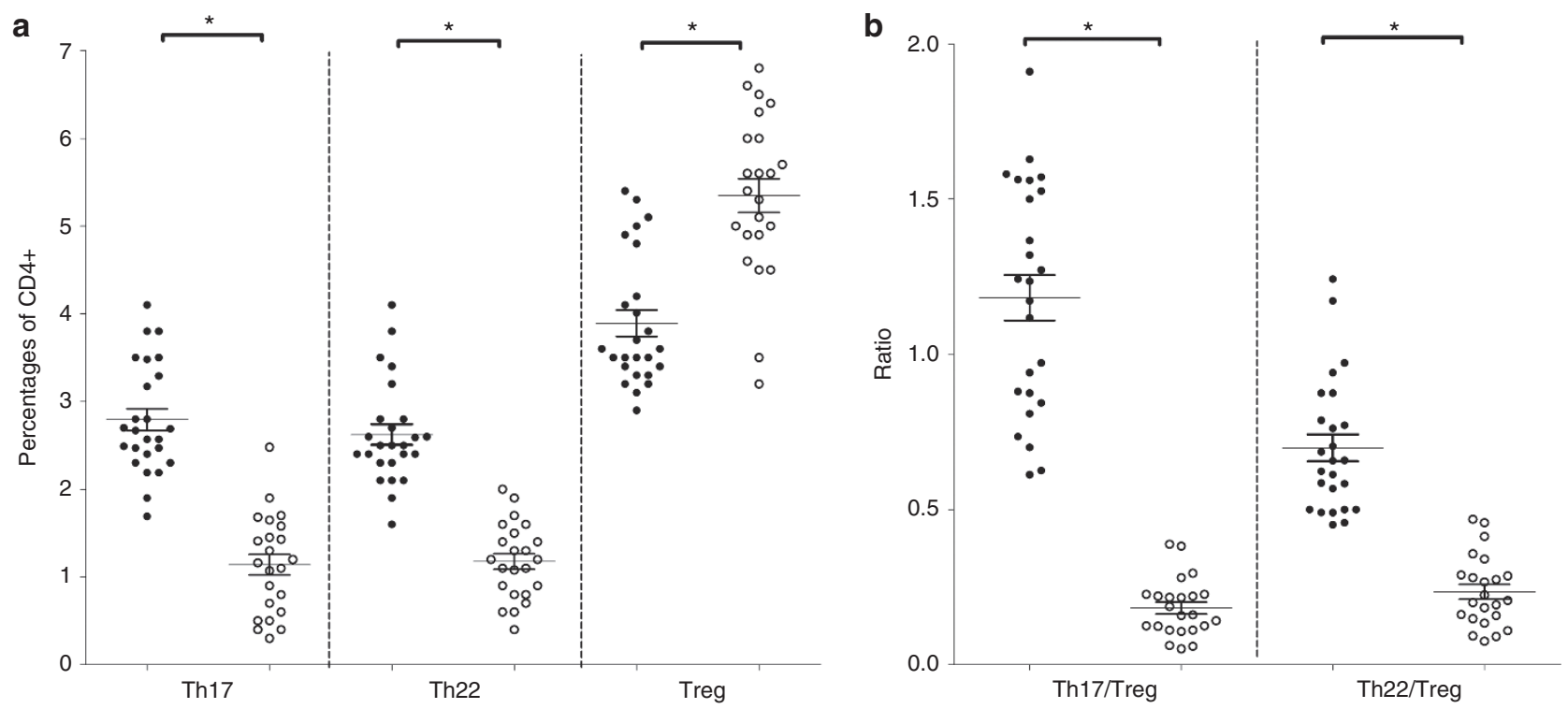

Figure 4. The impact of surgery on the peripheral distribution of CD4+T-lymphocyte subset. Filled circles $(0)$ represent data before surgery $(n=25)$ and empty circles (o) represent data obtained 6 mo after surgery $(n=23)$. The effects of surgery on the proportion of Th17, Th22, and Treg were presented (a). The changes of the peripheral Th17/Treg ratio $(1.18 \pm 0.36$ vs. $0.18 \pm 0.09, P<0.001)$ and Th22/Treg ratio $(0.70 \pm 0.19$ vs. $0.24 \pm 0.11, P=0.004)$ were shown (b). The statistical data were shown in Table 4. The horizontal lines represent the median values and error bars represent standard errors of means (SEM). ${ }^{*} P<0.05$. 
a<smiles>[Z]C[As]</smiles>

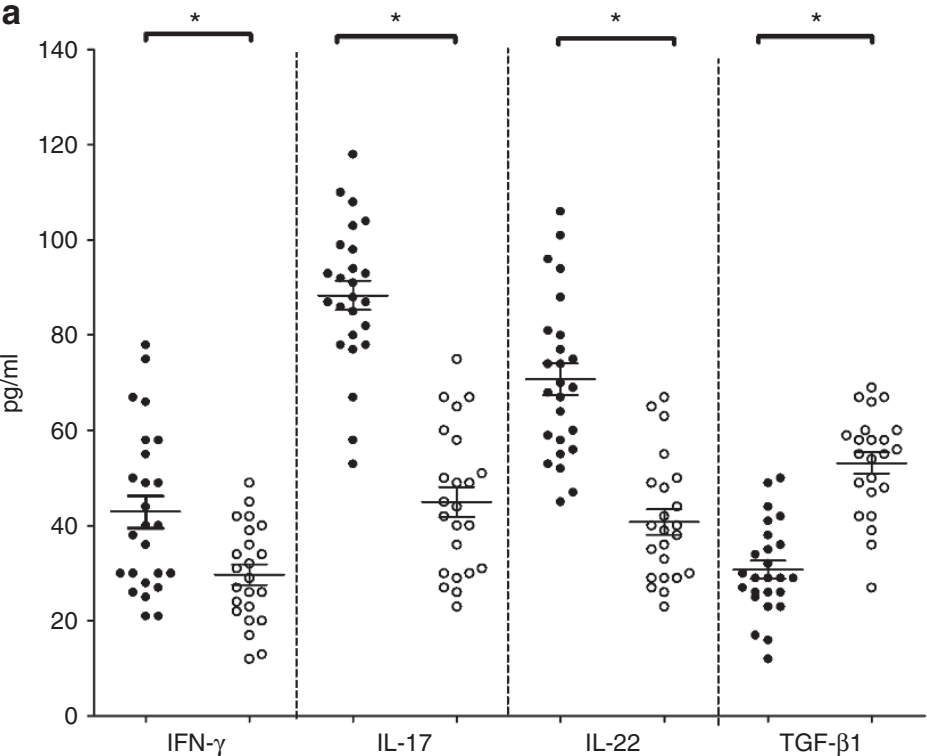

b

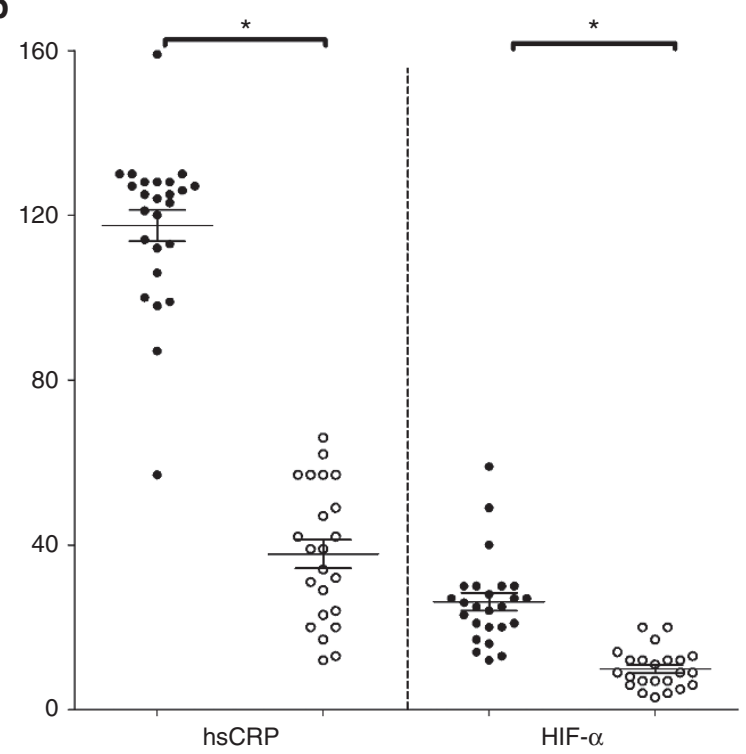

Figure 5. The impact of surgery on the peripheral expression of related cytokines and proinflammatory parameters. Filled circles ( $(\bullet)$ represent data before surgery $(n=25)$ and empty circles (o) represent data obtained 6 mo after surgery $(n=23)$. The changes of each Th subset-related cytokines (a) and inflammatory markers (b) were shown. The statistical data were shown in Table 4. The horizontal lines represent the median values and error bars represent SEM. ${ }^{*} P<0.05$. hsCRP, hypersensitive C-reactive protein.

SDB children. The reversibility of lymphocyte activation and cytokine explosion after adenotonsillectomy further support a direct effect of OSAS on systemic immunity independent of obesity. Steiropoulos et al. (20) demonstrated that nasal continuous positive airway pressure therapy selectively decreased the peripheral CD4+T-lymphocyte counts in OSAS patients. In brief, specific therapies for sleep apnea, either surgery or nasal continuous positive airway pressure, relieve the obstructive symptoms and also correct the immune imbalance underlying the long-term complications of OSAS.

\section{Limitations}

Some considerations must be acknowledged. First, we excluded obese children, which is a major confounder in most studies of OSAS. It suggests that the elevated CRP levels correlate with adipose tissue mass and obesity rather than OSAS. Indeed, obesity is also viewed as an inflammatory disorder and associated with a high risk for OSAS-induced morbidity (21). Second, we did not explore proliferation or function of lymphocyte subsets in vitro. Indeed, a previous report showed an enhanced proliferation of lymphocytes and increased concentration of cytokines in tonsils derived from OSAS children (22). Independent of these considerations, our findings comprehensively depicted the regional and systemic pattern of $\mathrm{CD} 4^{+} \mathrm{T}-$-lymphocyte subsets in the context of pediatric OSAS. Moreover, we investigated their clinical relevance and prognostic significance, all of which deserve further exploration.

\section{Conclusion}

In summary, our results indicate a direct association of the peripheral Th17/Treg imbalance with the repetitive IHR, subsequent inflammation and the severity of OSAS. Further studies to determine the threshold of Th17/Treg ratio as a valuable index in the clinical diagnosis and treatment response evaluation in OSAS would be of considerable interest. The molecular mechanisms that lead to an increased Th17/Treg ratio in response to IHR and the potential role of HIF-1 in this context should be explored in greater detail. It may provide greater insight into the pathological consequences of OSAS, and be essential to formulate strategies for long-term treatment and prevention in childhood.

\section{METHODS}

\section{Subjects and Protocol}

Consecutive children ( $4 \leq$ age $\leq 12$ y) who underwent tonsillectomy (with or without adenoidectomy) for SDB between January 2009 and December 2012 were eligible for enrollment. All the children had signs and symptoms of a sleep disturbance, including snoring, mouth breathing, and witnessed breath holding, for at least 3 mo. The following factors were indications for adenotonsillectomy (23): (i) adenotonsillar hypertrophy (tonsils $>2+$ and/or adenoids $>1+$ ), (ii) symptoms of SDB $>3$ nights per week, and (iii) AHI $>1$ episode per hour of sleep.

It is unethical to obtain tonsil samples in children who do not undergo adenotonsillectomy, and not all PS children generally need adenotonsillectomy. Only some PS children require surgery to eliminate obstructive symptoms due to hypertrophic tonsils and adenoids, frequent snoring, and a disturbed quality of life. This PS subpopulation is suitable for comparisons with OSAS.

We prospectively identified SDB children and allocated them into two groups based on the presence of an AHI $>1$ (namely OSAS) to identify distinctive characteristics for OSAS. The enrolled children were carefully screened and matched for age, sex, tonsillar size, BMI $z$ score, and ethnicity.

Healthy nonobese children without SDB-related symptoms were recruited as control subjects during the research period. All the control children had an $\mathrm{AHI} \leq 1$ during sleep and no adenotonsillar hypertrophy.

The following exclusion criteria were used for all participants: (i) recurrent tonsillitis or otitis media; (ii) allergy or atopic condition, such as asthma or allergic rhinitis; (iii) craniofacial abnormalities; (iv) chronic pulmonary, cardiovascular, neuromuscular, or genetic 
disorders; (v) symptoms or signs of acute or chronic inflammatory disorders; (vi) any known immunodeficiency; and (vii) use of nasal corticosteroids, systemic steroids, or antibiotics within 6 mo before surgery. The patients with sleep disorders, such as upper airway resistance syndrome, central sleep apnea syndrome, periodic limbs movement, or narcolepsy, were also removed from the final analyses.

All participants were required to complete a clinical evaluation within 2 wk before surgery and 6 mo after adenotonsillectomy that included an obstructive sleep apnea 18-Item quality-of-life questionnaire (OSA-18), Epworth sleepiness scale (ESS) evaluation, blood assay, and overnight PSG. The Institutional Review Board of Sun Yat-sen University approved the study, and informed consent was obtained from the legal caregiver of each participant.

\section{Data Collection}

Current and previous medical histories were recorded, and an overall physical examination was performed for each participant. Anthropometric data (age, sex, BMI $z$ score) and SDB-related symptoms were recorded. Obesity was defined as a BMI in the $>95$ th percentile for gender and age, which corresponded to a BMI $z$ score of 1.64 , based on normal values of Chinese children using CDC 2000 growth standards and online software. Therefore, nonobese children were included if their BMI $z$ score was $<1.64$.

Notably, a preoperative evaluation of tonsil size but not adenoid size is better correlated with symptoms and PSG-measured OSAS severity $(24,25)$. Tonsil size in children was graded as 0 to 4 suggested by Brodsky (26), according to an assessment performed with an endoscope by otolaryngology clinicians within $1 \mathrm{wk}$ before surgery. All data were collected using a specially designed case report form, and the data were manually double-entered into a Microsoft Excel master sheet to build our database. Another researcher checked all forms for errors.

\section{Overnight Polysomnography}

A sleep medicine physician interpreted the PSG results. PSG was conducted using the Embla-Monet 32 Sleep System (Embla, Broomfield, $\mathrm{CO})$ and manually scored by a single registered technologist as described in our previous work $(6,27,28)$. Hypopnea was defined as a decrease of $\geq 30 \%$ in thoracoabdominal motion associated with a fall in baseline oxygen saturation of $>4 \%$. The severity of hypoxia was assessed by calculating the lowest oxygen saturation (nadir $\mathrm{SaO}_{2}$ ) and the percentage of total sleep time spent with an oxygen saturation at $\mathrm{SaO}_{2}<90 \%$. The obstructive apnea-hypopnea index (OAHI) was defined as the total number of obstructive and mixed apneas and obstructive hypopneas per hour of total sleep time, and SDB severity was defined as follows $(29,30)$ : OAHI $\leq 1$ event $/ \mathrm{h}=\mathrm{PS}$; OAHI $>1-\leq 5$ events $/ \mathrm{h}=$ mild OSA; OAHI > 5-10 events $/ \mathrm{h}=$ moderate OSA; OAHI $>10$ events $/ \mathrm{h}=$ severe OSA. Subjects with central sleep apnea were not included in this study.

\section{Tissue Collection and Preparation}

Tonsil tissues were placed in ice-cold phosphate-buffered saline (PBS) immediately after surgical excision and transferred rapidly to the laboratory for further processing. The tonsils were washed in saline solution to eliminate any possible blood contamination and divided into halves. One half of the tissue sample was fixed in $4 \%$ buffered formalin, routinely processed for subsequent immunohistochemical assessment. The remaining tonsillar tissue was manually dissected, screened, and washed to separate tonsillar mononuclear cells. The cell pellet was resuspended in Roswell Park Memorial Institute medium (all from Gibco BRL, Gaithersburg, MD) for use in a quantitative realtime polymerase chain reaction (RT-qPCR) or flow cytometry.

\section{Blood Samples and PBMC Isolation}

Blood samples were obtained in the morning after PSG following an overnight fast. Peripheral whole blood samples $(15-20 \mathrm{ml})$ were collected in collection tubes containing $0.2 \mathrm{ml}$ sodium heparin. PBMCs were isolated from whole blood using Ficoll-Hypaque density centrifugation according to the manufacturer's instructions.

\section{Flow Cytometric Analysis}

Intracellular cytokines were studied using FCM to identify cytokineproducing cells. Briefly, PBMCs or tonsillar mononuclear cells were
Table 6. Primers used for quantitative RT-PCR

\begin{tabular}{lc}
\hline Genes & \multicolumn{1}{c}{ Primers } \\
\hline T-bet & \\
Sense & 5'-GCGCCAGGAAGTTTCATTT-3' \\
Antisense & 5'-CATTCTGGTAGGCAGTCACG-3' \\
GATA-3 &
\end{tabular}

GATA-3
Sense

Antisense

ROR $\gamma t$

Sense

Antisense

AhR

Sense

Antisense

Foxp3

Sense

Antisense suspended at a density of $2 \times 10^{6}$ cells $/ \mathrm{ml}$ in complete culture medium (Roswell Park Memorial Institute 1640 medium supplemented with $100 \mathrm{U} / \mathrm{ml}$ penicillin, $100 \mu \mathrm{g} / \mathrm{ml}$ streptomycin, $2 \mathrm{mmol} / \mathrm{l}$ glutamine, $10 \%$ heat-inactivated fetal calf serum; Gibco BRL, Gaithersburg, $\mathrm{MD})$. Cultures were stimulated with phorbol myristate acetate (50 ng/ $\mathrm{ml})$ plus ionomycin $(1 \mu \mathrm{mol} / \mathrm{l})$ for $4 \mathrm{~h}$ in the presence of monensin (500 ng/ml; all from Alexis Biochemicals, San Diego, CA). An incubator was set at $37^{\circ} \mathrm{C}$ under a $5 \% \mathrm{CO}$ environment. The contents of each well were transferred to 5 -ml sterile tubes after $4 \mathrm{~h}$ of culture and centrifuged at $1,500 \mathrm{rpm}$ for $5 \mathrm{~min}$.

The following monoclonal antibodies were used in this study: PerCP-eFluor 710-conjugated anti-CD8, APC-conjugated anti-CD3, APC-conjugated anti-CD25, FITC-conjugated anti-IFN $\gamma$, Alexa Fluor 488-conjugated anti-IL17A, PE-conjugated anti-IL4, PE-conjugated anti-IL22, and PE-conjugated anti-FoxP3. Isotype controls were given to enable correct identification and confirm antibody specificity. All antibodies were from eBioscience (San Diego, CA). Cells were incubated with surface antibodies for $15 \mathrm{~min}$ at room temperature, and the cells were stained with intracellularly antibodies using the Fix/Perm Buffer Kit (eBioscience, San Diego, CA) for fixation and permeabilization according to the manufacturer's protocol.

The prevalence of Th17 cells in PBMCs was expressed as a ratio of CD4 ${ }^{+}$IFN- $\gamma$ IL-22 $\mathrm{IL}^{-17^{+}} / \mathrm{CD}^{+} \mathrm{T}$ cells. Th 22 was defined as $\mathrm{CD} 4^{+} \mathrm{IFN}-$ $\gamma$ IL-17 IL-22+ T cells to exclude Th1 or Th17 cells. The prevalence of Treg cells was expressed as a ratio of $\mathrm{CD} 4^{+} \mathrm{CD} 25^{+} \mathrm{Foxp}^{+} / \mathrm{CD} 4^{+} \mathrm{T}$ cells. Stained cells were analyzed with flow cytometric analysis using a FACScan flow cytometer (Becton Dickinson, Franklin Lakes, NJ) equipped with CELLQest Pro 5.2 software (BD Biosciences, PharMingen). The data were analyzed using FlowJo (TreeStar, Ashland, OR). Lymphocyte subsets are expressed as percentages of the total amount of $\mathrm{CD} 4^{+}$cells isolated from tonsillar cellular suspensions or peripheral blood.

\section{Measurement of Plasma Cytokines and hsCRP Using ELISA}

To verify the results of flow cytometry, we also quantified representative cytokines of CD4 ${ }^{+} \mathrm{T}-$ lymphocyte lineages by ELISA: Th1 (IFN- $\gamma$ ), Th2 (IL-4), Th22 (IL-22), Th17 (IL-17), and Treg (TGF- $\beta_{1}$ ). The plasma levels of related cytokines were measured using ELISA following the manufacturer's instructions for each product (ELISA kits, all from R\&D system, Minneapolis, MN). The minimal detectable concentrations were $2 \mathrm{pg} / \mathrm{ml}$ for IL-17, $9 \mathrm{pg} / \mathrm{ml}$ for IL-22, $0.7 \mathrm{pg} / \mathrm{ml}$ for IL-6, and $5 \mathrm{pg} / \mathrm{ml}$ for TGF- $\beta_{1}$. The serum levels of hsCRP were determined with particle-enhanced immunoturbidimetry (Beijing O\&D Biotech Company, Cox Bio China, Beijing, China). The lower limit of detection for hsCRP was $0.06 \mathrm{mg} / \mathrm{l}$. All samples were measured in triplicate. 


\section{Real-Time Quantitative RT-PCR}

The expression of representative transcription factors of each lineage were assessed by qPCR: Th1 (T-bet), Th2 (GATA-3), Th22 (AhR), Th17 (ROR $\gamma \mathrm{t}$ ), and Treg (FOXP3). Total RNA was extracted from PBMCs and tonsillar mononuclear cells using TRIzol extraction (Invitrogen, Carlsbad, CA) and quantified using a spectrophotometer based on the absorbance A260/A280 ratio. The primer pairs used are listed in Table 6. Quantitative real-time PCR were performed using TaqMan one-step RT-PCR reagents kits (Applied Biosystems, Foster City, CA) according to the manufacturer's instructions. Thermocycling was run in an ABI Prism 7900 Sequence Detection System (Applied Biosystems) with the following conditions: $30 \mathrm{~min}$ at $50{ }^{\circ} \mathrm{C}$, $10 \mathrm{~min}$ at $95^{\circ} \mathrm{C}, 40 \mathrm{cycles}$ of $15 \mathrm{~s}$ at $95^{\circ} \mathrm{C}$, and $1 \mathrm{~min}$ at $60^{\circ} \mathrm{C}$.

\section{Statistical Analysis}

The values in the tables and figures unless stated otherwise are expressed as the means \pm SD. Student's $t$-test or one-way ANOVA was used when appropriate to compare continuous variables, and $\chi^{2}$ test was used for categorical variables. Spearman's correlation analyses were conducted to examine potential associations between systematic distributions of the CD4 ${ }^{+} \mathrm{T}-$ lymphocyte subsets and other variables. Univariate and stepwise multivariate linear regression analyses were conducted with the Th17/Treg ratio as a dependent variable in relation to AHI and other covariates. A $P$ value $<0.05$ indicated significance. Statistical analyses were performed using a commercial software package (SPSS, version 15)

\section{STATEMENT OF FINANCIAL SUPPORT}

This study was supported by the Science and Technology Planning Project of Guangdong Province, China (No.2011B061300008).

Disclosures: None of the participating institutions and authors has conflicts of interest regarding the study.

\section{REFERENCES}

1. Marcus CL, Brooks LJ, Draper KA, et al.; American Academy of Pediatrics. Diagnosis and management of childhood obstructive sleep apnea syndrome. Pediatrics 2012;130:e714-55.

2. Ryan S, Taylor CT, McNicholas WT. Selective activation of inflammatory pathways by intermittent hypoxia in obstructive sleep apnea syndrome. Circulation 2005;112:2660-7.

3. Garvey JF, Taylor CT, McNicholas WT. Cardiovascular disease in obstructive sleep apnoea syndrome: the role of intermittent hypoxia and inflammation. Eur Respir J 2009;33:1195-205.

4. Liu H, Zhang T, Ye J, et al. Tumor-infiltrating lymphocytes predict response to chemotherapy in patients with advance non-small cell lung cancer. Cancer Immunol Immunother 2012;61:1849-56.

5. Cheng X, Yu X, Ding YJ, et al. The Th17/Treg imbalance in patients with acute coronary syndrome. Clin Immunol 2008;127:89-97.

6. Ye J, Liu H, Zhang G, et al. The treg/th17 imbalance in patients with obstructive sleep apnoea syndrome. Mediators Inflamm 2012;2012:815308.

7. Barbi J, Pardoll D, Pan F. Metabolic control of the Treg/Th17 axis. Immunol Rev 2013;252:52-77.

8. Verhulst SL, Aerts L, Jacobs S, et al. Sleep-disordered breathing, obesity, and airway inflammation in children and adolescents. Chest 2008;134: 1169-75.

9. Dyugovskaya L, Lavie P, Lavie L. Lymphocyte activation as a possible measure of atherosclerotic risk in patients with sleep apnea. Ann N Y Acad Sci 2005;1051:340-50.

10. Zhang L, Li JM, Liu XG, et al. Elevated Th22 cells correlated with Th17 cells in patients with rheumatoid arthritis. J Clin Immunol 2011;31:606-14.
11. Zhang L, Li YG, Li YH, et al. Increased frequencies of Th22 cells as well as Th17 cells in the peripheral blood of patients with ankylosing spondylitis and rheumatoid arthritis. PLoS One 2012;7:e31000.

12. Truchetet ME, Brembilla NC, Montanari E, Allanore Y, Chizzolini C. Increased frequency of circulating Th22 in addition to Th17 and Th2 lymphocytes in systemic sclerosis: association with interstitial lung disease. Arthritis Res Ther 2011;13:R166.

13. Tan HL, Gozal D, Wang Y, et al. Alterations in circulating T-cell lymphocyte populations in children with obstructive sleep apnea. Sleep 2013;36: 913-22.

14. Tan HL, Gozal D, Samiei A, et al. T regulatory lymphocytes and endothelial function in pediatric obstructive sleep apnea. PLoS One 2013;8:e69710.

15. Lages CS, Suffia I, Velilla PA, et al. Functional regulatory T cells accumulate in aged hosts and promote chronic infectious disease reactivation. J Immunol 2008;181:1835-48.

16. Bollinger T, Bollinger A, Naujoks J, Lange T, Solbach W. The influence of regulatory $\mathrm{T}$ cells and diurnal hormone rhythms on $\mathrm{T}$ helper cell activity. Immunology 2010;131:488-500.

17. Kim J, Bhattacharjee R, Khalyfa A, et al. DNA methylation in inflammatory genes among children with obstructive sleep apnea. Am J Respir Crit Care Med 2012;185:330-8.

18. Yuan G, Nanduri J, Bhasker CR, Semenza GL, Prabhakar NR. Ca2+/ calmodulin kinase-dependent activation of hypoxia inducible factor 1 transcriptional activity in cells subjected to intermittent hypoxia. J Biol Chem 2005;280:4321-8.

19. Dang EV, Barbi J, Yang HY, et al. Control of T(H)17/T(reg) balance by hypoxia-inducible factor 1. Cell 2011;146:772-84.

20. Steiropoulos P, Kotsianidis I, Nena E, et al. Long-term effect of continuous positive airway pressure therapy on inflammation markers of patients with obstructive sleep apnea syndrome. Sleep 2009;32:537-43.

21. Gozal D, Kheirandish-Gozal L. Childhood obesity and sleep: relatives, partners, or both?-a critical perspective on the evidence. Ann N Y Acad Sci 2012;1264:135-41.

22. Kim J, Bhattacharjee R, Dayyat E, et al. Increased cellular proliferation and inflammatory cytokines in tonsils derived from children with obstructive sleep apnea. Pediatr Res 2009;66:423-8.

23. Kaditis AG, Ioannou MG, Chaidas K, et al. Cysteinyl leukotriene receptors are expressed by tonsillar $\mathrm{T}$ cells of children with obstructive sleep apnea. Chest 2008;134:324-31.

24. Toros SZ, Noşeri H, Ertugay CK, et al. Adenotonsillar hypertrophy: Does it correlate with obstructive symptoms in children? Int J Pediatr Otorhinolaryngol 2010;74:1316-9.

25. Granzotto EH, Aquino FV, Flores JA, Lubianca Neto JF. Tonsil size as a predictor of cardiac complications in children with sleep-disordered breathing. Laryngoscope 2010;120:1246-51.

26. Brodsky L. Modern assessment of tonsils and adenoids. Pediatr Clin North Am 1989;36:1551-69.

27. Ye J, Liu H, Zhang G, Huang Z, Huang P, Li Y. Postoperative respiratory complications of adenotonsillectomy for obstructive sleep apnea syndrome in older children: prevalence, risk factors, and impact on clinical outcome. J Otolaryngol Head Neck Surg 2009;38:49-58.

28. Ye J, Liu H, Li Y, Liu X, Zhu JM. Increased serum levels of C-reactive protein and matrix metalloproteinase-9 in obstructive sleep apnea syndrome. Chin Med J (Engl) 2007;120:1482-6.

29. Katz ES, Greene MG, Carson KA, et al. Night-to-night variability of polysomnography in children with suspected obstructive sleep apnea. J Pediatr 2002;140:589-94.

30. Jackman AR, Biggs SN, Walter LM, et al. Sleep disordered breathing in early childhood: quality of life for children and families. Sleep 2013;36:1639-46. 